\title{
Force Constants of Cu Crystals from Diffuse Neutron Scattering Measurement
}

\author{
T. Sakuma ${ }^{{ }^{\star}}$, S.R. Mohapatra ${ }^{1}$, H. Uehara ${ }^{1}$, R. Sakai ${ }^{1}$, Xianglian $^{2}$, \\ H. Takahashi ${ }^{3}$, N. Igawa ${ }^{4}$ and K. Basar ${ }^{5}$ \\ ${ }^{1}$ Institute of Applied Beam Science, Ibaraki University, Mito 310-8512, Japan \\ ${ }^{2}$ College of Physics and Electronics Information, Inner Mongolia University for the Nationalities, Tongliao 028043, China \\ ${ }^{3}$ Institute of Applied Beam Science, Ibaraki University, Hitachi 316-8511, Japan \\ ${ }^{4}$ Quantum Beam Science Directorate, Japan Atomic Energy Agency, Tokai 319-1195, Japan \\ ${ }^{5}$ Faculty of Mathematics and Natural Sciences, Bandung Institute of Technology, Bandung 40132, Indonesia
}

\section{ARTICLE INFO}

\section{Article history:}

Received 12 October 2010

Received in Revised form 22 October 2010

Accepted 16 December 2010

Keywords:

Diffuse scattering

Force constants

Neutron scattering

Thermal diffusion

Thermal vibrations

\begin{abstract}
A B S T R A C T
Diffuse neutron scattering measurement on Cu crystals was performed at $10 \mathrm{~K}$ and $300 \mathrm{~K}$. Oscillatory forms were observed in the diffuse scattering intensities. The observed diffuse scattering intensities are analyzed by including the correlation effects among thermal displacements of atoms in the theory. Using the values of correlation effects among neighboring atoms and the values of Debye-Waller temperature parameter, force constants among first, second and third nearest neighboring atoms have been evaluated. The result of correlation effects in $\mathrm{Cu}$ crystals are compared to that of ionic crystal and semiconductor. The relation between correlation effects and the inter-atomic distance is not depending much on the crystal binding types.
\end{abstract}

(C) 2010 Atom Indonesia. All rights reserved

\section{INTRODUCTION}

Diffuse scattering measurement using X-ray and neutron is a versatile technique to investigate lattice dynamical properties in various kinds of ordered and disordered solids. An early study on disordered $\alpha$-AgI type solid electrolytes showed anomalously strong and oscillatory diffuse scattering intensities [1]. The temperature dependence of the strong diffuse scattering intensity was explained by the Debye-Waller temperature parameters, short-range order of cations and the values of the correlation effects among thermal displacements of atoms. The presence of correlation effects among first, second and third nearest neighboring atoms has also been noticed in ordered ionic crystals and semiconductors [2,3] as it is indicative from the analysis of diffuse scattering patterns in these materials. In the present study, the correlation effects are investigated in metal crystals from diffuse neutron scattering measurement. Interatomic force constants of metal crystals are estimated using values of correlation effects.

\footnotetext{
* Corresponding author.

E-mail address: sakuma@mx.ibaraki.ac.jp ( T. Sakuma )
}

\section{EXPERIMENTAL METHODS}

Neutron scattering measurements at temperature $10 \mathrm{~K}$ and $300 \mathrm{~K}$ have been performed on powder $\mathrm{Cu}$ crystals using HRPD (High Resolution Powder Diffractometer) installed at JRR-3 in Japan Atomic Energy Agency. HRPD is an angular-dispersive type diffractometer with 64 counters and has high resolution performance that could separate diffuse scattering intensities from Bragg lines in diffraction patterns. Powder sample was set in a vanadium container of $10 \mathrm{~mm}$ in diameter. Incident neutron wavelength of $1.823 \AA$ that monochromatized by Ge (331) was used and the data were collected for 1 day in the $2 \theta$ range from 30 to $140^{\circ}$ with step angle $0.05^{\circ}$.

\section{RESULTS AND DISCUSSION}

The observed neutron diffraction intensities of $\mathrm{Cu}$ are shown in Fig. 1. The maximum observed intensities of Bragg lines are about 60,000. When we magnify the base region of diffraction pattern in Fig. 1, weak diffuse scattering intensities are revealed as displayed in Fig. 2. Some clear peaks of oscillatory diffuse scattering were observed at $300 \mathrm{~K}$ at $2 \theta$ around 60 and $120^{\circ}$. The difference of the diffuse scattering intensity between $10 \mathrm{~K}$ and $300 \mathrm{~K}$ is about 100 counts. 


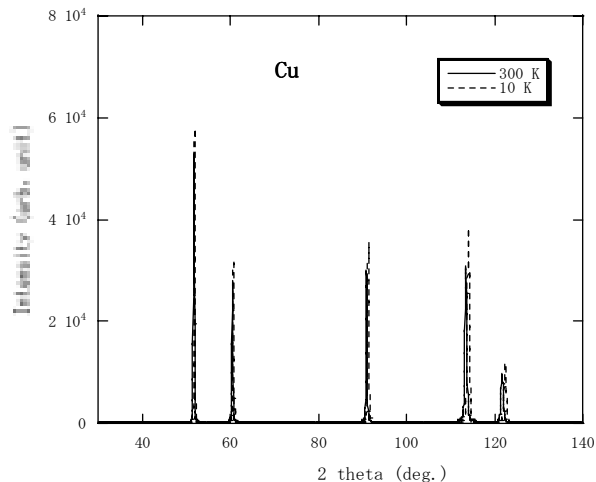

Fig. 1. Observed neutron diffraction intensity of $\mathrm{Cu}$ at $10 \mathrm{~K}$ and $300 \mathrm{~K}$.

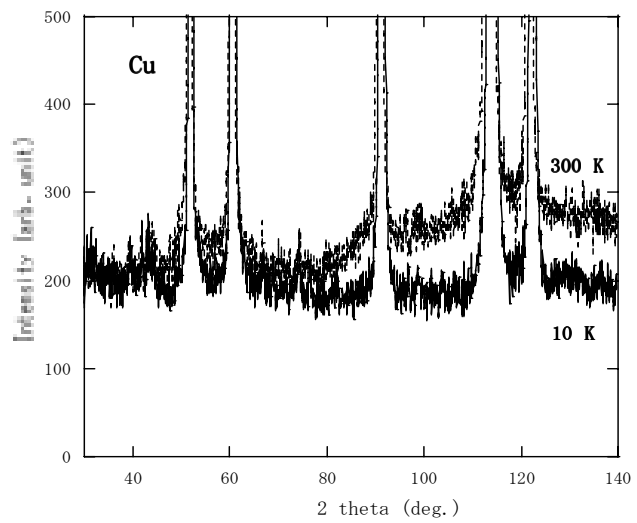

Fig. 2. Diffuse neutron scattering intensities of $\mathrm{Cu}$ at $10 \mathrm{~K}$ and $300 \mathrm{~K}$.

Rietveld analysis has been performed on the neutron diffraction data of $\mathrm{Cu}$ using RIETAN-2000 [4]. The crystal of $\mathrm{Cu}$ was assumed to have fcc type structure with the space group $F m \overline{3} m$. The calculated values of lattice constant and Debye-Waller temperature parameter of $\mathrm{Cu}$ are $a=3.598 \AA$, $B=0.052 \AA^{2}$ at $10 \mathrm{~K}$ and $a=3.607 \AA, B=0.469 \AA^{2}$ at $300 \mathrm{~K}$. The parameters like number of neighboring atoms $Z$, inter-atomic distance $r$ and correlation effects $\mu$ used for generating the diffuse scattering intensities are shown in Table 1.

Table 1. Number of neighboring atoms $Z$, inter-atomic distance $r$ and correlation effects $\mu$ of $\mathrm{Cu}$ at $10 \mathrm{~K}$ and $300 \mathrm{~K}$.

\begin{tabular}{lllclc}
\hline & \multicolumn{3}{c}{$10 \mathrm{~K}$} & \multicolumn{3}{c}{$300 \mathrm{~K}$} \\
\cline { 2 - 6 } & $Z$ & $r(\AA)$ & $\mu$ & $r(\AA)$ & $\mu$ \\
\hline 1st & 12 & 2.544 & 0.33 & 2.550 & 0.61 \\
2nd & 6 & 3.598 & 0.20 & 3.607 & 0.40 \\
3rd & 24 & 4.404 & 0.05 & 4.415 & 0.08 \\
\hline
\end{tabular}

The intensity of diffuse neutron scattering including the correlation effects among thermal displacements of atoms is expressed as follows [5];

$$
\begin{gathered}
I_{\mathrm{D}}=I_{\mathrm{o}} K N_{\mathrm{o}} \sum_{s} n b_{s} b_{s}^{*}\left[1-\exp \left(-2 M_{s}\right)\right] \\
+I_{\mathrm{o}} K N_{\mathrm{o}} \sum_{s} \sum_{s^{\prime}} n b_{s} b_{s^{\prime}}^{*}\left[\exp \left\{-\left(M_{s}+M_{s^{\prime}}\right)\left(1-\mu_{s s^{\prime}}\right)\right\}\right. \\
\left.-\exp \left\{-\left(M_{s}+M_{s^{\prime}}\right)\right\}\right] Z_{s s^{\prime}} \frac{\sin (Q r)}{Q r}+I_{\mathrm{o}} K N_{\mathrm{o}} \sum_{s} \sigma_{\text {inc }}+C
\end{gathered}
$$

where $b_{s}$ is nuclear scattering length for atom in site $s, I_{0} K$ a constant depending on the experimental condition, $N_{\mathrm{o}}$ the number of the unit cell in unit volume, and $Z$ the number of sites belonging to the $s$ 'th neighbor around an sth site. $n$ corresponds to the number of atoms per unit cell. Two sites $s$ and $s^{\prime}$ are apart by the distance $r \cdot \exp (-M)$ $\left(=\exp \left\{-B(\sin \theta / \lambda)^{2}\right\}\right)$ is Debye-Waller factor of the atom. $\sigma_{\text {inc }}$ is incoherent scattering cross-section of atoms. The constant $C$ is added for the corrections of background noise. The oscillatory form is expressed as $\sin (Q r) / Q r$, where $Q$ is equal to $4 \pi \sin \theta / \lambda$. The correlation effects among the thermal displacements of atoms $\mu$ are written as;

$$
\mu_{s s^{\prime}}=2\left\langle\Delta \boldsymbol{r}_{s} \cdot \Delta \boldsymbol{r}_{s^{\prime}}\right\rangle /\left(\left\langle\Delta \boldsymbol{r}_{s}^{2}\right\rangle+\left\langle\Delta \boldsymbol{r}_{s^{\prime}}^{2}\right\rangle\right)
$$

Debye-Waller temperature parameter $B$ is defined by $B=8 \pi^{2}\left\langle\Delta r^{2}\right\rangle$, where the deviation from an equilibrium position is shown by $\Delta \boldsymbol{r}$. The values of the correlation effects among the thermal displacements of $s$ and $s^{\prime}$ atoms is 0 in the absence of any correlation.

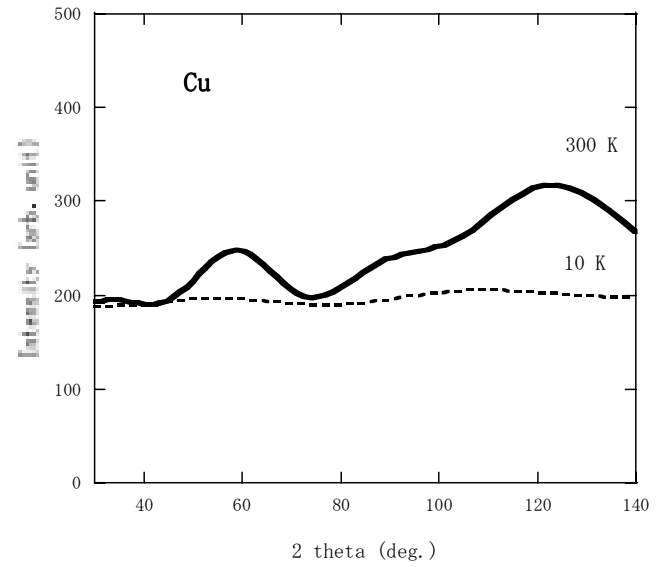

Fig. 3. Calculated diffuse scattering intensities of $\mathrm{Cu}$ at $10 \mathrm{~K}$ and $300 \mathrm{~K}$.

The diffuse scattering intensity of $\mathrm{Cu}$ was calculated from the values of Debye-Waller temperature parameter, number of neighboring atoms, inter-atomic distances and correlation effects at $10 \mathrm{~K}$ and $300 \mathrm{~K}$ in Table 1 . The calculated diffuse 
scattering intensities at $10 \mathrm{~K}$ and $300 \mathrm{~K}$ are shown in Fig. 3. It is found that the observed oscillatory diffuse scattering intensity of $\mathrm{Cu}$ in Fig. 2 is matching qualitatively with the calculated pattern.

As the value of correlation effects of first nearest neighboring atoms is greater than that of second and third nearest neighboring atoms, the oscillatory scheme in the diffuse scattering intensity of $\mathrm{Cu}$ is mainly coming from the contribution of first nearest neighboring atoms. To confirm further, we assumed three different values of the correlation effects among thermal displacements of first nearest neighboring atoms as $0.1,0.5$ and 0.9 and calculated the diffuse scattering intensities of $\mathrm{Cu}$ at $10 \mathrm{~K}$ and $300 \mathrm{~K}$ as plotted in Fig. 4 and 5, respectively. In this calculation, the correlation effects of second and third nearest neighboring atoms were presumed to be 0 . From Fig. 5 it is found that the oscillatory scheme of the diffuse scattering intensity at $300 \mathrm{~K}$ varies largely with the change of the correlation effects.

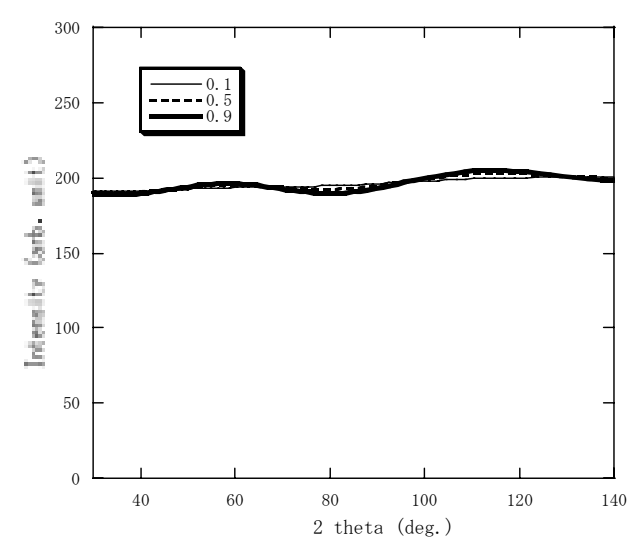

Fig. 4. Diffuse scattering intensities with the change of correlation effects among first nearest neighboring atoms at $10 \mathrm{~K}$.

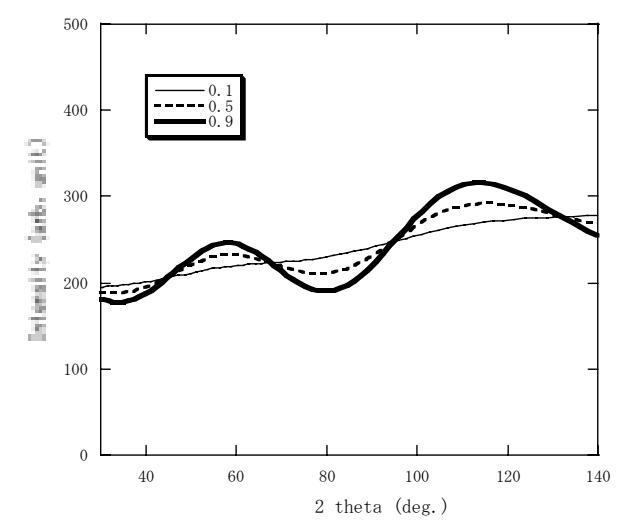

Fig. 5. Diffuse scattering intensities with the change of correlation effects among first nearest neighboring atoms at $300 \mathrm{~K}$.
The values of correlation effects of $\mathrm{Cu}$ are plotted with that of $\mathrm{ZnSe}$ and $\mathrm{KBr}[6]$ as shown in Fig. 6. It is observed that the correlation effects decrease with the increase of inter-atomic distance and increase with temperature. The value of the correlation effects is about 0.7 for the inter-atomic distance $r \sim 2.5 \AA$ and about 0.4 for $r \sim 4 \AA$ near room temperature. The correlation effects are not depending significantly on the type of crystal structure and crystal binding near room temperature.

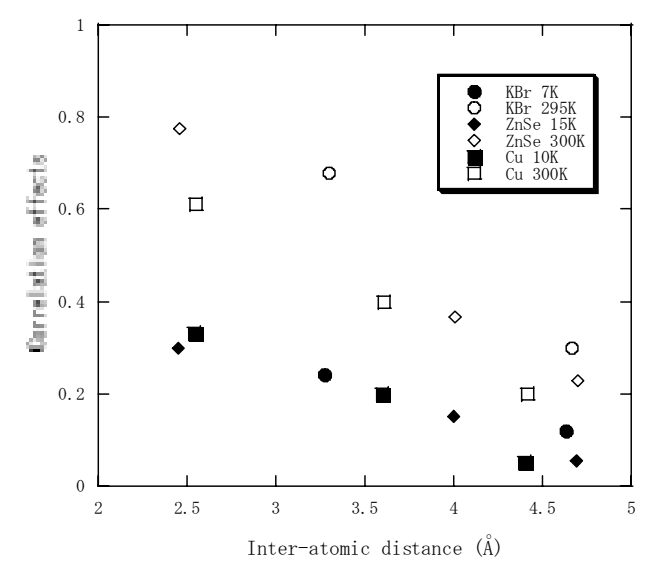

Fig. 6. Correlation effects of $\mathrm{Cu}, \mathrm{ZnSe}$ and $\mathrm{KBr}$.

From the values of correlation effects $\mu$ among $s$ and $s$, atoms and Debye-Waller temperature parameter $B_{s}$ and $B_{s}$, we could estimate the values of force constants $\alpha_{s s^{\prime}}$ among first, second and third nearest neighboring atoms [2] as follows;

$$
k_{\mathrm{B}} T / \alpha_{s s^{\prime}}=\left(B_{s}+B_{s^{\prime}}\right)\left(1-\mu_{s s^{\prime}}\right) / 8 \pi^{2} .
$$

The estimated values of force constants among first, second and third nearest neighboring atoms of $\mathrm{Cu}$, are 5.58, 3.63 and $2.37 \mathrm{eV} / \AA^{2}$ at $300 \mathrm{~K}$, respectively. The force constant for first nearest neighboring atoms of $\mathrm{Cu}$ at $300 \mathrm{~K}$ is comparable to that of $\mathrm{ZnSe}$ (6.16) but greater than that of $\mathrm{KBr}(1.45)[3,7]$. However, the values of correlation effects among thermal displacements of first nearest neighboring atoms in $\mathrm{Cu}, \mathrm{ZnSe}$ and $\mathrm{KBr}$ at room temperature are about $\sim 0.7$. This indicates that the variation in their inter-atomic force constants is mostly due to the variation in DebyeWaller temperature factors.

\section{CONCLUSIONS}

Oscillatory form of the diffuse neutron scattering intensity for $\mathrm{Cu}$ was explained on inclusion of correlation effects among thermal displacements of atoms in the theory. From the 
values of correlation effects and Debye-Waller temperature parameter, force constants among first, second and third nearest neighboring atoms were calculated. The relation between correlation effects and the inter-atomic distance did not depend much on the crystal binding types.

\section{ACKNOWLEDGMENTS}

This research was partially supported by the Ministry of Education, Science, Sports and Culture, Japan, Grant-in-Aid for Scientific Research (C), 21540314, 2010. One of the authors (X.) wishes to express her thanks for financial support to the Inner Mongolia Autonomous Region University, China (Grant No. NJ10114) and the Doctor-Master research fund of Inner Mongolia University for the Nationalities, China (Grant No. BS239).

\section{REFERENCES}

1. T. Sakuma, Bull. Electrochem. 11 (1995) 57.

2. T. Sakuma, Xianglian, S. Siagian, K. Basar, H. Takahashi, N. Igawa, and O. Kamishima, J. Thermal Anal. Cal. 99 (2010) 173.

3. K. Basar, S. Siagian, Xianglian, T. Sakuma, H. Takahashi and N. Igawa, Nuclear Instruments and Methods in Physics Research, A600 (2009) 237.

4. F. Izumi and T. Ikeda, Mater. Sci. Forum 198203 (2000) 321.

5. T. Sakuma, J. Phys. Soc. Jpn. 62 (1993) 4150.

6. K. Basar, Xianglian, T. Sakuma, H. Takahashi and N. Igawa, ITB J. Sci., 41 A (2009) 50.

7. T. Sakuma, Xianglian N. Shimizu, S.R. Mohapatra, N. Isozaki, H. Uehara, H. Takahashi, K. Basar, N. Igawa and O. Kamishima, Solid State Ionics, in: press, (DOI: 10.1016/j.ssi.2010.03.002 ). 